\title{
Les prises d'eau de l'aménagement de Gabi de la S.A. Energie Électrique du Simplon
}

\author{
Water intakes in the Gabi project \\ (S.A.) Énergie Électrique du Simplon
}

\author{
PAR \\ J. C. OTT \\ ET \\ INGÉNIEUR E.P.U.L. \\ SOUS-DIRECTEUR DE IAA SOCLETE GÉNÉRALE POUR I'INDUSTRIE,
} GENÈVE
La tendance à créer de vastes aménagements hydroélectriques, recueillant dans tout un réseau de vallées les eaux accumulées dans un site favorisé, conduit à exiger des performances accrues des prises d'eau tant au point de vue de leur rendement qu'au point de vue de leurs conditions d'exploitation. Le constructeur de celles-ci doit viser à satisfaire aux conditions suivantes :

- sécurité de fonctionnement,

- faible surveillance,

- évacuation des débits solides avec un minimum de pertes d'eau,

- coefficient de captage élevé.

Les auteurs décrivent des prises et les solutions variées adoptées pour satisfaire aux exigences mentionnées, notamment :

- une prise combinée avec dégraveur automatique,

- une prise avec dessableur combiné à l'intérieur du barrage de retenue,

- une prise tyrolienne de construction simplifiée, sans dégraveur.

Des considérations sur le fonctionnement des divers organes en cours d'exploitation sont développées, compte tenu des conditions de hante montagne dans lesquelles les prises sont placées. Une solution particulière de joint pour des canaax d'adduction préfabriqués en béton précontraint, résistant au gel et n'affaiblissant pas les éléments en béton est également décrite.
The present trend in creating hydro-electric power plants, collecting water from various valleys and concentrating it in a favorable site, leads to require increased performances from the water intakes, from the standpoint of efficiency as well as from the standpoint of their working conditions.

The constructor of such intakes seeks to satisf!I the following conditions:

- security in operation,

- low supervision requirements,

- evacuation of solid contents with a minimum of water losses,

- high collection coefficient.

The writers describe intakes and the various adopted solutions in order to satisfy the mentioned requirements, namely:

- an intake combined with an antomatic rocktrap,

- an intake with a sandtrap incorporated into the dam,

- a Tyrolian intake of a simplified construction, without any rocktrap.

Considerations in regard to the operation of the various elements during exploitation will be looked into further taking into account the high mountain conditions and altitude (about 5,000 feet) where the intakes will be installed. A. special solution for a joint for the power channels prefabricated in prestressed cement, frost-resistant, and which does not weaken the concrete elements, is also described.

\section{GENERALITÉS}

La mise en valeur des ressources hydroélectriques des Alpes conduit de plus en plus à envisager de vastes ensembles intéressant plusieurs vallées avec des galeries d'adduction très ramifiées, apportant l'eau souvent à très grande distance vers un site plus particulierement favorisé pour la création d'une retenue importante. 
Cette évolution caractéristique des grands aménagements modernes, tels que Grande Dixence, en Suisse, ou Roselend, en France, a conduit, d'une part, à la multiplication des points de captage et, d'autre part, à des exigences accrues quant au rendement, aux conditions d'exploitation et à l'économie des prises d'eau.

L'amélioration des performances de ces organes est une nécessité dont l'évidence s'impose autant que l'amélioration des performances de l'équipement des usines proprement dites.

Les longues galeries sont trop coûteuses pour que l'on puisse s'offrir le luxe de laisser déverser une partie des eaux qui ruissellent sur les bassins versants.
Il en résulte que le coefficient de captage (1) doit tendre vers une valeur qui se rapproche de 0,95 à 1 (prise d'Eggen 0,96), ce qui implique d'équiper les prises pour un rapport d'équipement voisin compris entre 1,5 à 2 fois le module des mois d'été.

Durant les débâcles printanières ou les orages le débit solıde est souvent important, si bien que des dispositions particulières doivent être prises pour prévenir l'obstruction des galeries ou l'usure anormale des ouvrages, voire des turbines, lorsqu'aucune retenue n'est interposée.

$$
\text { (1) } \frac{\text { volume annuel capté par la prise }}{\text { volume annuel total des apports }}
$$

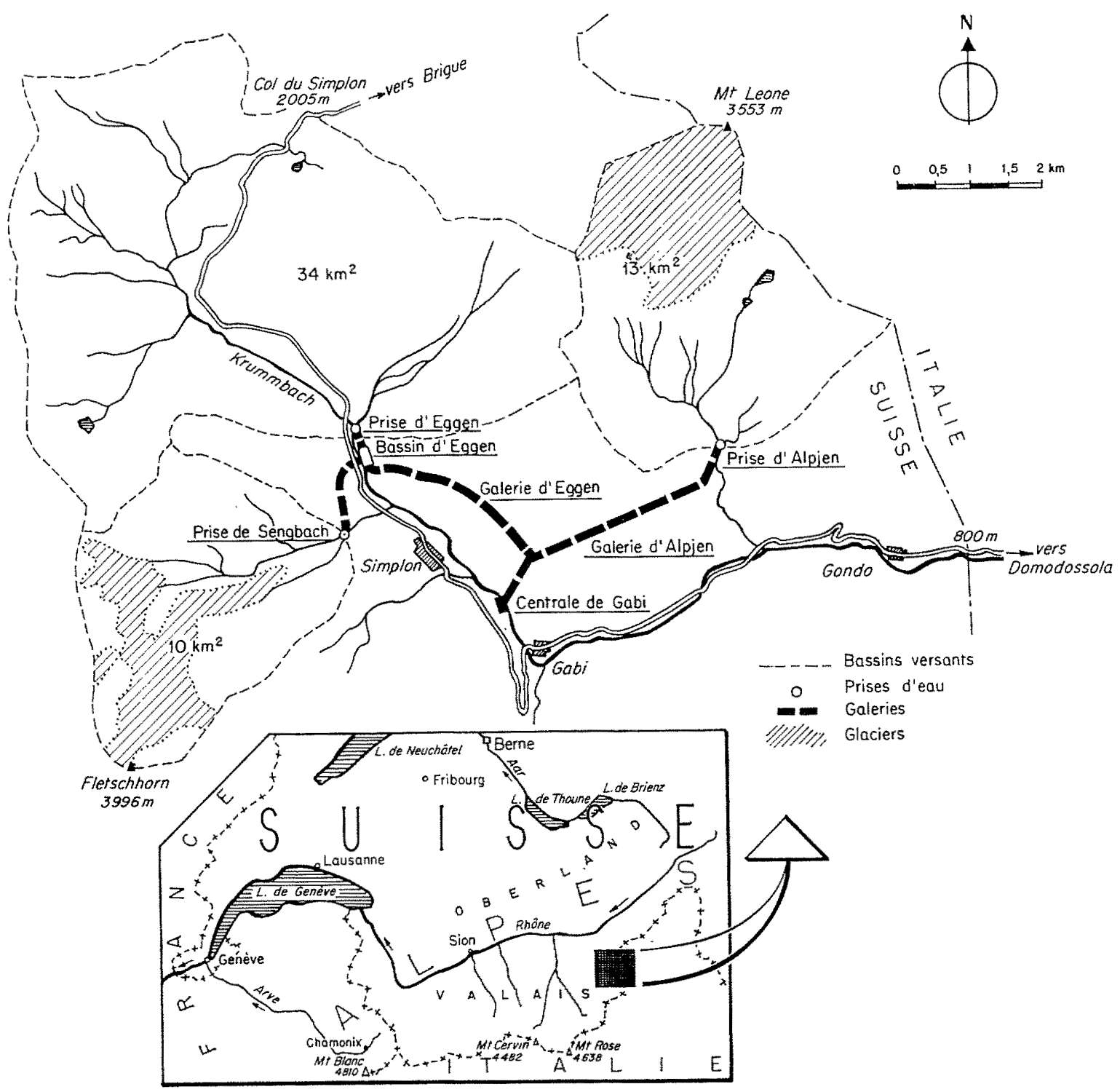

Fig. 1

Bassins versants des prises d'eau de l'aménagement de Gabi. 


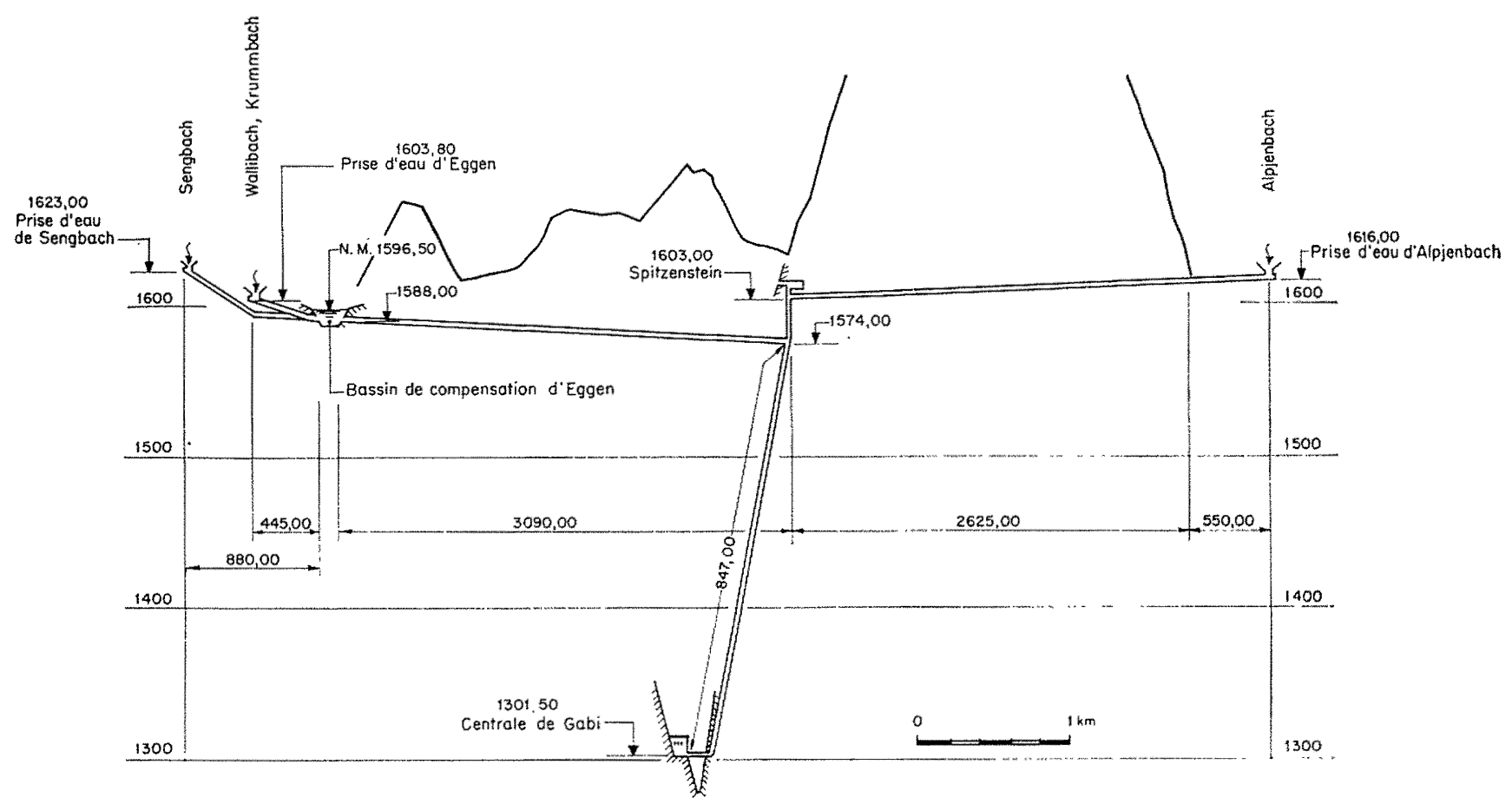

Fig. 2

Profil en long de l'aménagement.

Mais, plus le coefficient de captage est élcvé, plus s'accroissent les difficultés d'évacuer les matériaux solides de la prise et de les fairc transiter avec les débits restreints que l'on consent à perdre à cette fin. La correction du lit naturel des torrents à l'aval et parfois à l'amont des prises devient alors nécessaire.

Pour les prises éloignées et difficilement accessibles, l'éracuation des matériaux solides, qu'elle soit continue ou discontinue, doit être commandée par des mécanismes simples et automatiques, sans appel à une source d'énergie électrique.

Les multiples facteurs hydrologiques et géographiques, le caractère du cours d'eau, etc., déterminent le choix des caractéristiques de la prise; ils doivent être analysés avec soin, compte tenu de leur importance relative et des circonstances locales.

Bien que l'aménagement d'Energie Electrique du Simplon, dont les caractéristiques générales ont été publiées en 1958 dans le Bulletin de l'Association suisse des Electriciens, $n^{\circ} 9$, soit modeste, il comporte des prises de types variés dont les dispositions parfois originales présentent un certain intérêt dans le cadre de l'évolution générale mentionnéc plus haut.

Le schéma général de l'aménagement (fig. 1 et fig. 2) a été réalisé en trois étapes :
I) Usine de Gondo ( $36 \mathrm{MW}$ ) avec prise du Krumbach et du Lagginbach. Bassin de compensation à Serra;

II) Usine de Gabi (12 MW) avec les prises d'Eggen et de l'Alpjenbach. Bassin de compensation journalier à Eggen;

III) Prise du Sengbach, avec adduction au bassin d'Eggen.

\section{Conditions topographiques.}

L'élargissement de la vallée du Simplon, à Eggen, formée ici par des alluvions compactes, était tout indiqué pour y placer le bassin de compensation de la centrale de Gabi; au sud de cette petite plaine la roche est affleurante à l'emplacement choisi.

Pour la prise d'Eggen on a choisi un site aussi proche que possible du bassin de compensation, de facon à réduire le coût de la conduite d'amenée. Elle a, de ce fait, un fonctionnement en régime mixte: lorsque le bassin est à demi rempli, le tronçon inférieur de la conduite est complètement noyé, alors que le tronçon supérieur est à écoulement libre.

A Alpjen, l'emplacement favorable à une prise d'eau se situait à une cote nettement supérieure 

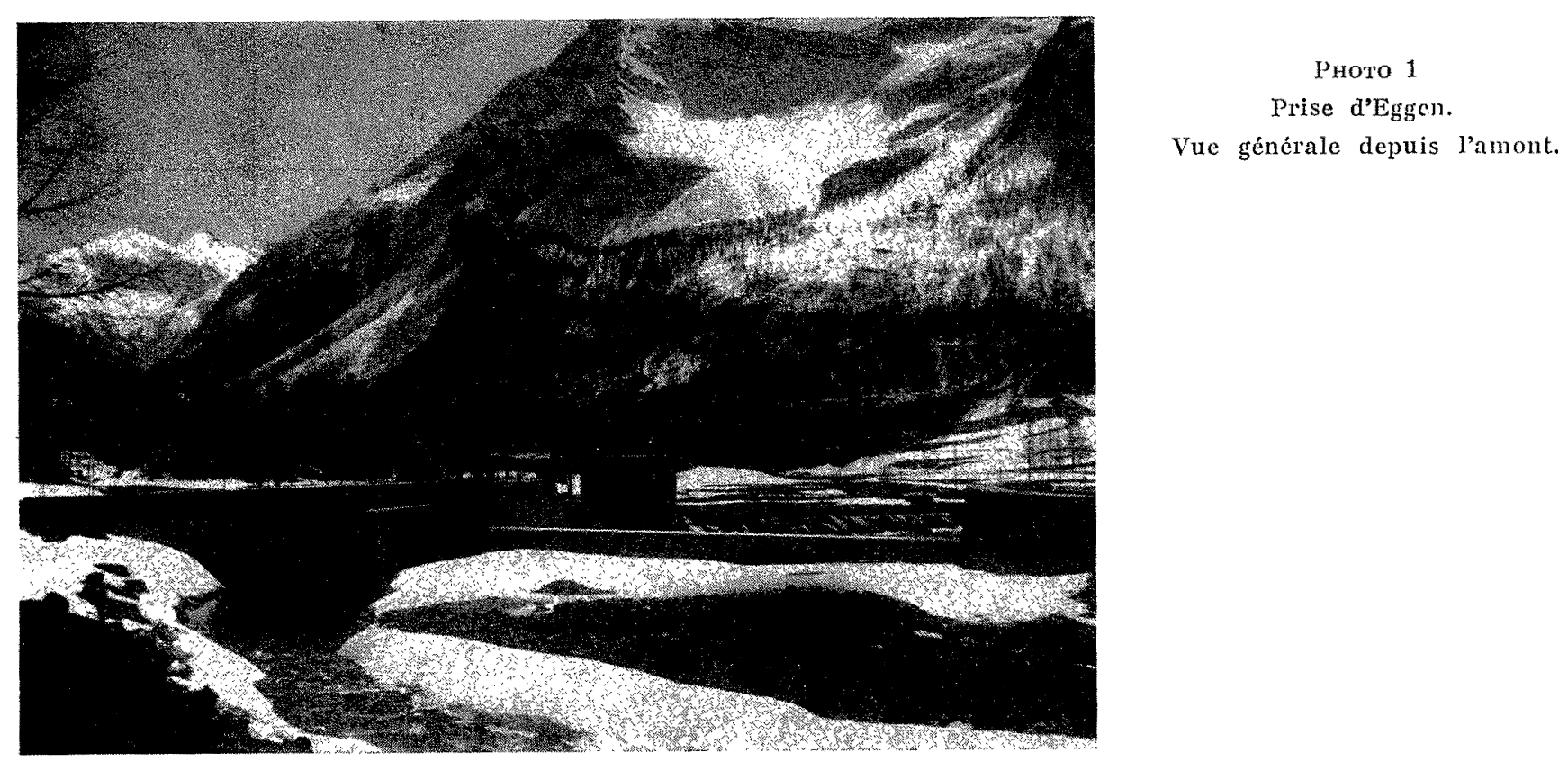

à la cote moyenne de la galerie d'amenée en charge d'Eggen. Pour cette raison, on décida de faire aboutir la galerie d'amenée d'Alpjen au sommet de la chambre d'équilibre, ce qui permit de la maintenir à écoulement libre et de supprimer le revêtement, à l'exclusion du radier qui est bétonné.

L'extrémité amont de la galerie d'Alpjen aurait débouché dans une épaisse couche morainique si on l'avait implantée au niveau de la prise. En la déplaçant vers l'aval, pour éviter cet inconvénient, il devint indispensable de réaliser une conduite de raccordement entre la prise et la galerie d'amenée.

La prise du Sengbach se trouve dans des conditions tout à fait différentes des prises susmentionnées. Elle est située sur un torrent, émissaire d'un vaste glacier dont le front s'appuie sur un éboulement assez récent; ce terrain sauvage est parsemé de grands blocs, d'éboulis et de maigres buissons.

\section{Conditions hydrologiques et météorolo- giques.}

Le climat du versant sud du Simplon diffère notablement de celui du nord des Alpes; les précipitations ne sont en général pas provoquées par le passage de dépressions dérivant d'ouest en est, en provenance de l'Océan, mais le plus souvent par des dépressions immobilisées dans le bassin méditerranéen; il en rẻsulte que, contrairement à ce qui se passe lorsque les Préalpes déchargent progressivement les fronts humides, les pluies se concentrent au pied des Alpes, là où les chaînes se redressent brutalement de toute leur hanteur. Des chutes de neige atteignant $2 \mathrm{~m}$ ne sont pas rares; $350 \mathrm{~mm}$ de pluie ont été mesurés pendant une journée exceptionnelle (automine 1958 à Varzo, altitude $530 \mathrm{~m}$ ).

Des crues extraordinairement brutales peuvent endommager, voire détruire, les ouvrages de prises, à moins qu'ils ne soient construits de façon exceptionnellement massive et résistante.

L'ingénieur-projeteur se trouve ainsi devant une alternative :

- Prévoir des ouvrages coûteux absolument sûrs et indestructibles;

ou :

- Envisager des ouvrages relativement vulnérables, mais à meilleur marché, quitte à ce qu'ils soient détruits par une crue exceptionnelle.

Cette deuxième possibilité peut se justifier pour des ouvrages de haute montagne, notamment dans le cas où leur coût d'établissement est à la limite de la rentabilité. La prise du Sengbach a été construite selon cette conception.

Quant aux prises d'eau d'Eggen et d'Alpjen, elles peuvent être considérées comme indestructibles et c'est la raison pour laquelle l'observateur non informé peut les juger surdimensionnées et massives.

Les prises sus-mentionnées sont également très différentes en ce qui concerne le régime hydrologique; alors que les torrents d'Eggen et d'Alpjen charrient relativement peu de matériaux solides vu la faible glaciation des bassins ver- 


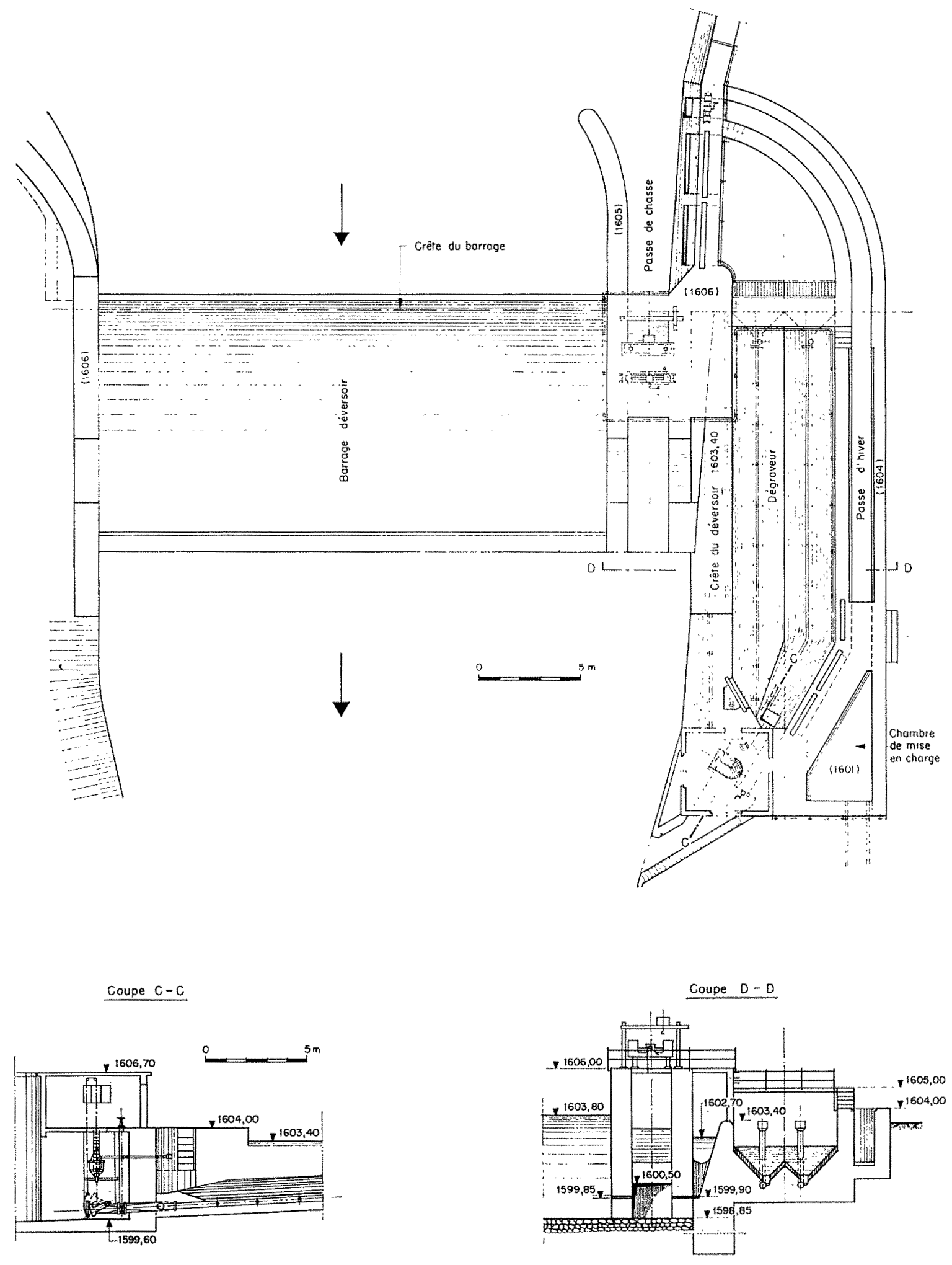

Fig. 3

Prise d'Eggen. 
sant, le torrent de Sengbach a, au contraire, un charriage important. Pour plus de précision, les caractéristiques du bassin sont indiquées cidessous :

\begin{tabular}{|c|c|c|c|c|c|c}
\hline Prise d'eau & $\begin{array}{c}\text { Bassin versant } \\
\mathrm{km}^{2}\end{array}$ & $\begin{array}{c}\% \\
\text { Glacicrs }\end{array}$ & $\begin{array}{c}\text { Apport d'eau } \\
\text { hiver (2) } \\
\text { en } 10^{6} \mathrm{~m}^{3}\end{array}$ & $\begin{array}{c}\text { Apport d'eau } \\
\text { été (2) } \\
\text { en } 10^{6} \mathrm{~m}^{3}\end{array}$ & $\begin{array}{c}\text { Apport d'eau } \\
\text { année } \\
\text { en } 10^{6} \mathrm{~m}^{3}\end{array}$ & $\begin{array}{c}\text { Rapport } \\
\text { d'équipement (3) }\end{array}$ \\
\hline Eggen $\ldots \ldots \ldots$ & 34 & 1 & 14,6 & 32,8 & 47,4 & 1,8 \\
Alpjen $\ldots \ldots \ldots$ & 15 & 28 & 3,1 & 18,1 & 21,2 & 2,0 \\
Sengbach $\ldots \ldots$ & 10 & 30 & 2,7 & 14,6 & 17,3 & 1,8
\end{tabular}

(2) Hiver : octobre-avril; été : mai-septembre.

(3) Rapport d'équipement $=\frac{\text { débit nominal de la prise }}{\text { débit moyen d'été }}$

Pour les prises d'Eggen el d'Alpjen, les dimensions des déversoirs de crue ont été calculées pour une crue maximale de $3 \mathrm{~m}^{3} / \mathrm{s} . \mathrm{km}^{2}$. Vu le type particulier de la prise du Sengbach, elle est susceptible de laisser passer tous les débits de crue.

Il semble à première vue paradoxal et exagéré d'équiper des prises à $4 \mathrm{~m}^{3} / \mathrm{s}$ (Eggen), 2슬 $\mathrm{m}^{3} / \mathrm{s}$ (Alpjen) et $1,8 \mathrm{~m}^{3} / \mathrm{s}$ (Sengbach), au total $8,3 \mathrm{~m}^{3} / \mathrm{s}$, pour une centrale dont le débit nominal est limité à $4,5 \mathrm{~m}^{3} / \mathrm{s}$.

Il y a cependant une raison à cela. Les périodes de fort débit sont décalées pour les trois prises. Pour le bassin versant d'Alpjen situé en plein sud, la fonte des neiges commence au mois de mars, même souvent en février, soit environ un mois avant celle du bassin versant d'Eggen.

Le bassin versant du Sengbach, par ses glaciers et son orientation, est soumis à des conditions différentes : forts débits à la fin de l'été, faibles débits en cas de temps pluvieux (4).

(4) En effet, les débits de cette prise, observés lors de l'été pluvieux et frais de 1960 , étaient relativement faibles.

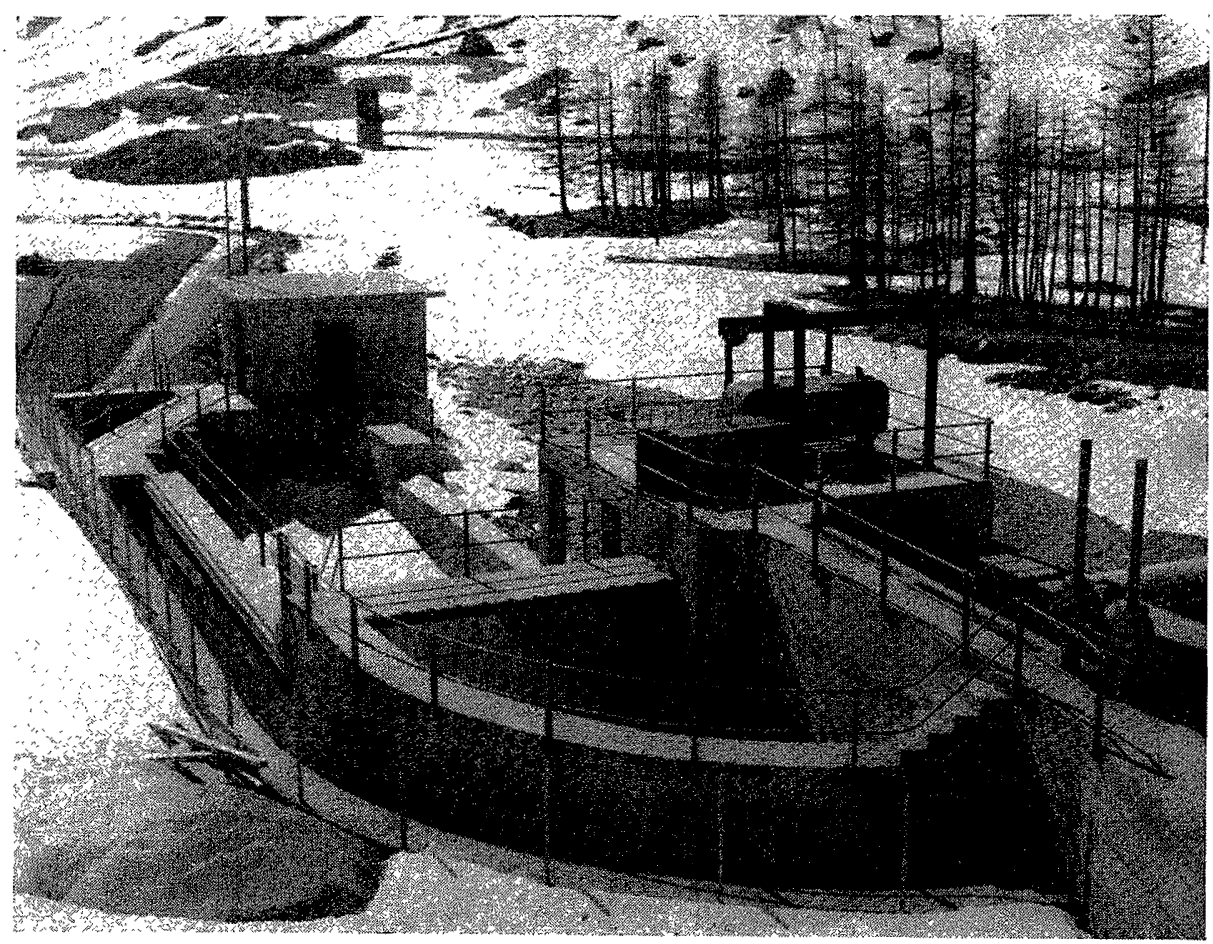

Pното 2

Prise d'Eggen. Le dégraveur (hors service).

L'eau passe par la prise et le chenal d'hiver visible à gauche. 
Il apparaît donc qu'il convient ainsi de ne pas projeter les prises avec des coefficients de captage identiques, mais bien de les adapter aux particularités des bassins versants, en cherchant à obtenir le maximum de rendement global.

\section{Description des ouvrages, fonctionnement des prises, observations.}

\section{a) Prise d'eau d'Eggen (fig 3) :}

La faible pente du cours du Krummbach, la proximité de la route du Simplon et l'obligation de ne pas perdre de chute imposaient un ouvrage relativement bas, du type latéral, comportant un barrage fixe de $25 \mathrm{~m}$ de largeur et $7 \mathrm{~m}$ de hauteur, avec passe de chasse. La passe de chasse comporte une vanne de fond et une vanne de surface pour évacuer les corps flottants. Une

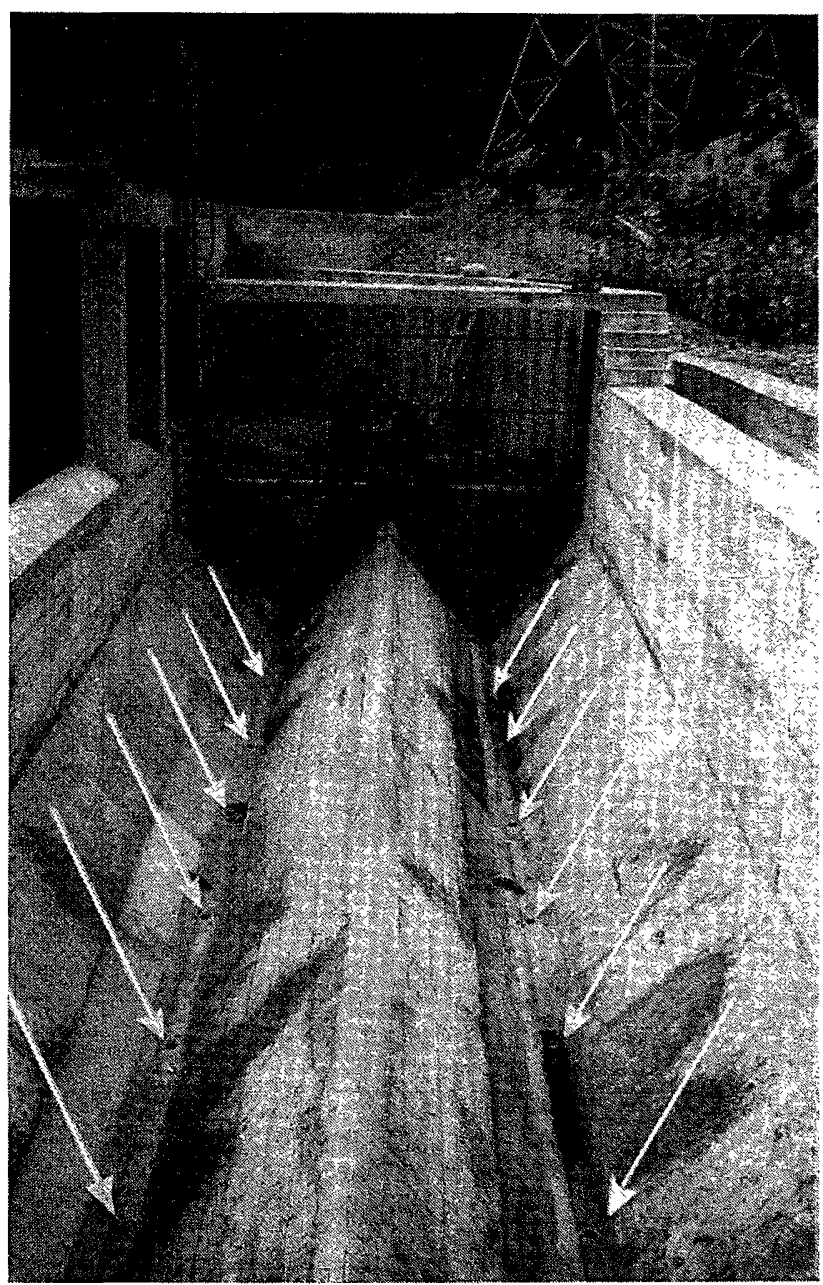

Pното 3

Prise d'Eggen. Intérieur du dégraveur. Trous du chenal d'évacuation des graviers (voir photo $\mathrm{n}^{\circ} 4$ ).

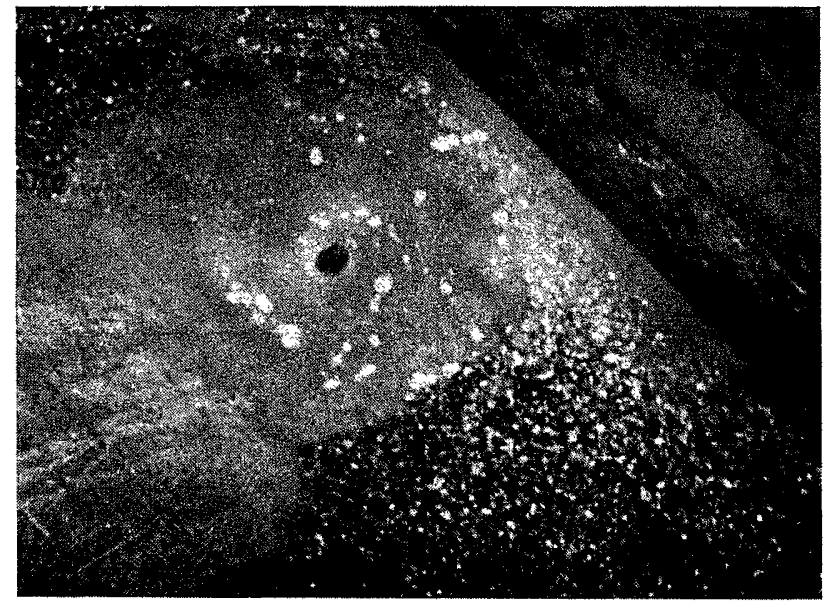

$a$

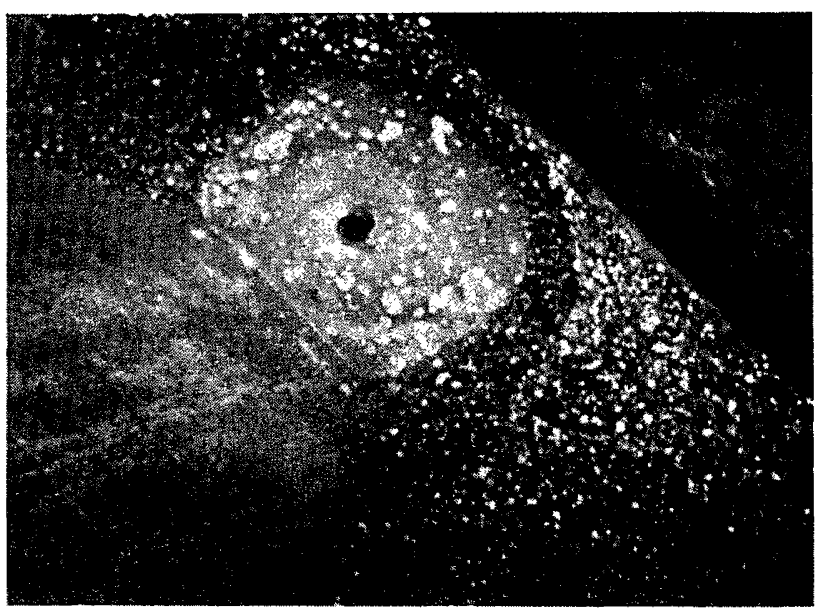

$b$

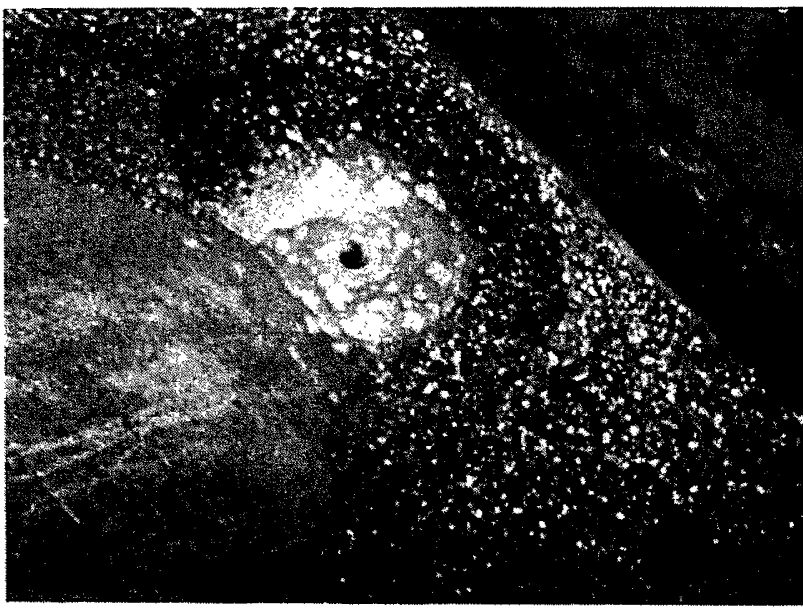

Photo 4

Prise d'Eggen.

Fonctionnement des trous d'évacuation du gravier. (Essai filmé)

Intervalle : environ deux secondes. 


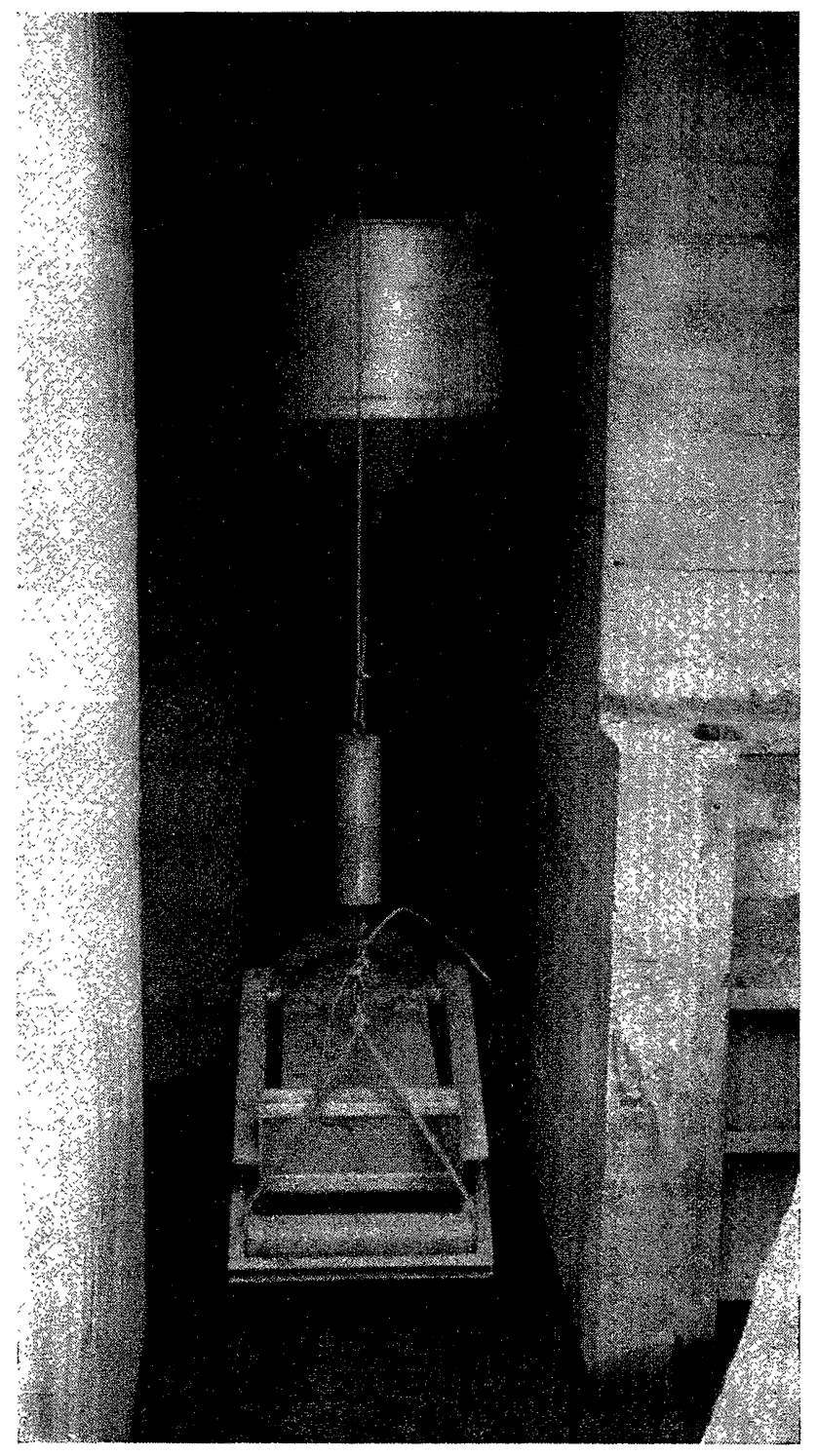

Pното 5

Prise d'Eggen.

Vanne secteur automatique (système Gianella) avec commande par contrepoids hydraulique dont on aperçoit la cuve.

petite prise d'hiver indépendante est également prévue.

On n'a pas jugé nécessaire ici de prévoir un véritable dessableur, mais simplement un dégraveur avec bassin court, le charriage n'étant important qu'en cas de crues exceptionnelles; en outre, les eaux, avant d'être turbinées, passent par le bassin d'Eggen où a lieu la décantation des sédiments fins.

Mais dans l'ignorance des sujétions éventuelles pour le curage du bassin, on jugea nécessaire de pousser le dégravage jusqu'à $1 \mathrm{~mm}$. On adopta pour la disposition des canaux de purge du dégraveur, le système breveté Gianella qui empêche, lors du remplissage du fond du bassin par les sédiments, que les conduits d'évacuation ne s'obstruent. Ce système comporte également une vanne automatique d'un type spécial.

L'ouverture de la vanne de purge est commandée automatiquement au moment où le débit dérivé dépasse une certaine cote (début du charriage) qu'on peut fixer librement. Depuis lors, d'autres systèmes basés sur la détection de l'engravement ont été développés.

Lorsque la cote d'amorçage est atteinte, une petite prise auxiliaire alimente un récipient suspendu à un câble qui, en se remplissant, descend et provoque l'ouverture d'une vannesecteur de fond du bassin de dégravage.

Le gravier déposé au fond des canaux est sucé par les trous du tuyau décrit plus haut, et il se forme une série d'entonnoirs qui s'agrandissent peu à peu jusqu'à ce que les trous du tuyau et le dégraveur soient complètement dégagés.

L'abaissement du niveau dans le dégraveur interrompt l'alimentation du récipient qui se vide par un trou calibré ouvert en permanence; la vanne de purge se ferme.

La purge fonctionne de façon continue tant que le débit est supérieur à la valeur fixée. Malheureusement, aucune grosse crue n'a provoqué un charriage important qui permette de corroborer les excellents résultats obtenus lors de l'essai du dispositif avec du gravier.

La liaison prise d'Eggen — bassin d'Eggen est réalisée au moyen d'une conduite en tuyaux préfabriqués. Comme nous l'avons dit plus haut, cette conduite fonctionne en régime mixte.

\section{b) Prise d'Alpjen :}

Le site de la prise d'Alpjen forme une petite plaine à faible pente, se terminant par un goulet bordé de part et d'autre par des terrains morainiques; en aval, la vallée présente des pentes latérales assez rapides, où il est difficile d'implanter des ouvrages. Le rocher n'affleure nulle part dans le lit. Il parut judicieux de placer la prise à l'entrée du goulet; mais il était difficile à cet endroit d'exécuter des ouvrages latéraux (dessableurs, etc.). Par ailleurs, les accès précaires de chantier (un téléphérique monocâble, pas de pistes pour jeep) ne permettaient pas l'organisation de bétonnages importants. On choisit donc un type d'ouvrage fortement évidé, présentant un volume de béton minimum. On en vint ainsi tout naturellement à l'idée dê faire un barrage de section triangulaire à parements amont et aval inclinés, contenant tous les organes de la prise et le dessableur. En effet, un dessableur était ici indispensable, l'eau dérivée d'Alpjen étant conduite directement aux turbines de Gabi sans bassin de mise en charge. 

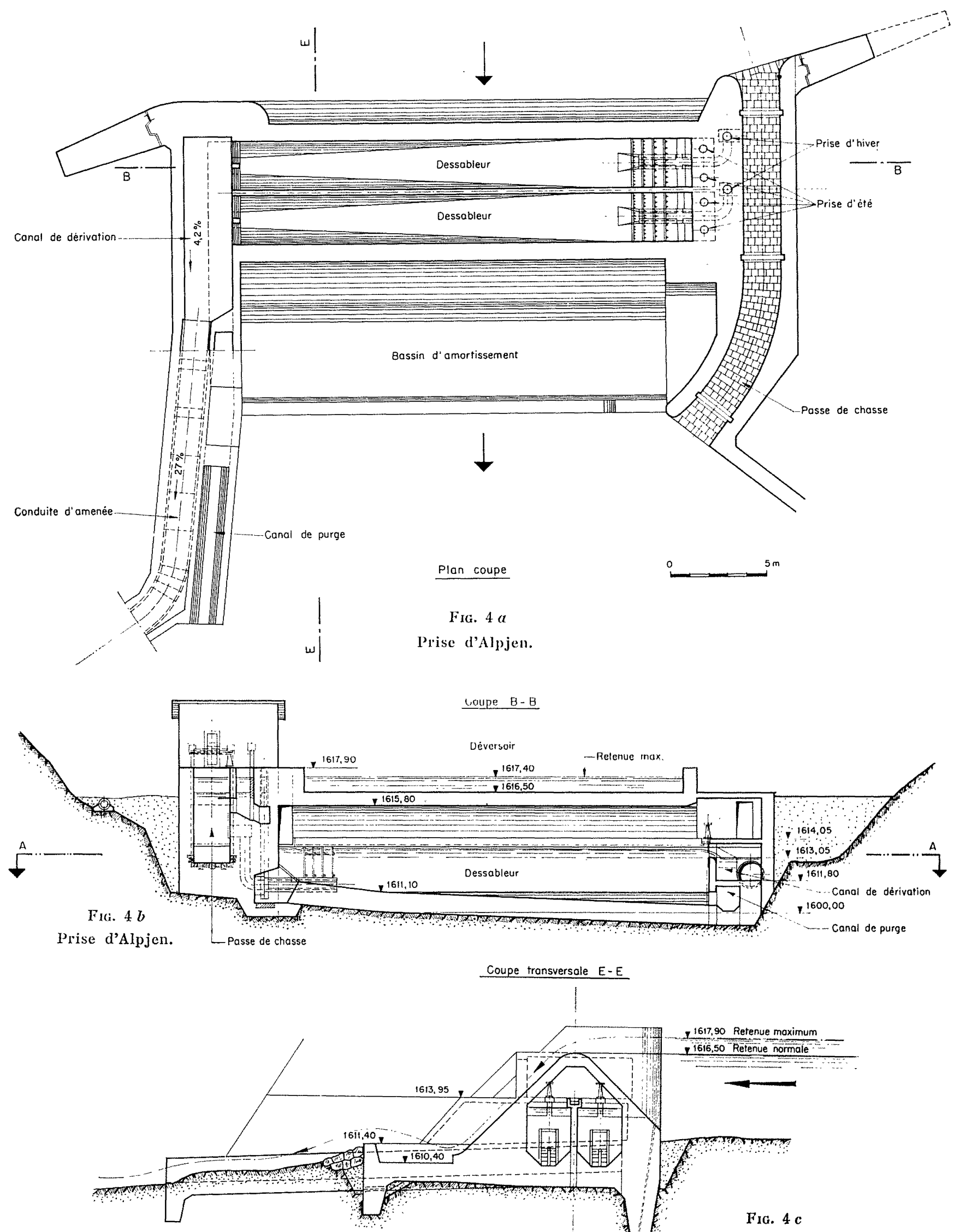

Prise d'Alpjen. 
Les divers organes furent disposés de facon originale comme le montre la figure 4 .

Sur la rive gauche se trouve une passe de chasse avec vanne de fond et vanne de surface. Dans le bajoyer de la rive gauche qui forme l'extrémité amont du dessableur sont piquées quatre conduites aboutissant dans le bassin de décantation à l'intérieur du barrage. Les plans d'eau des deux bassins de décantation se trouvent à $3,0 \mathrm{~m}$ en contrebas de la crête $\mathrm{du}$ barrage. Grâce à cette dénivellation, le débit qui pénètre dans la prise varie peu lorsque la lame déversante augmente d'épaisseur. En effet, ce débit est une fonction de la chute dont la hauteur de lame ne représente qu'une petite fraction. On avait intérêt également à limiter le débit de prise en cas de crue, de façon à ne pas introduire inutilement des matériaux dans le dessableur, ce qui aurait nécessité des purges trop fréquentes (le surveillant n'habite que six mois par an à proximité de l'ouvrage). Pour y parvenix, les «pipes» de prises sont munies en leur partie inférieure de manchettes de réglage du débit. Elles débouchent dans une fosse de tranquillisation de l'écoulement avant l'entrée dans le bassin de dégravage.

Il résulte de cette disposition que le dégravage n'est pas très poussé; heureusement, la plaine située à l'amont de la prise favorise une décantation préliminaire.

La prise fonctionne depuis 1957. Les constatations suivantes ont été faites lors des premières années de fonctionnement :

- Le dessablage est suffisant pour prévenir l'obstruction du siphon situé à l'aval sur la conduite d'amenée;

- En période de crue, il suffit de faire fonctionner les purges une fois par jour; par
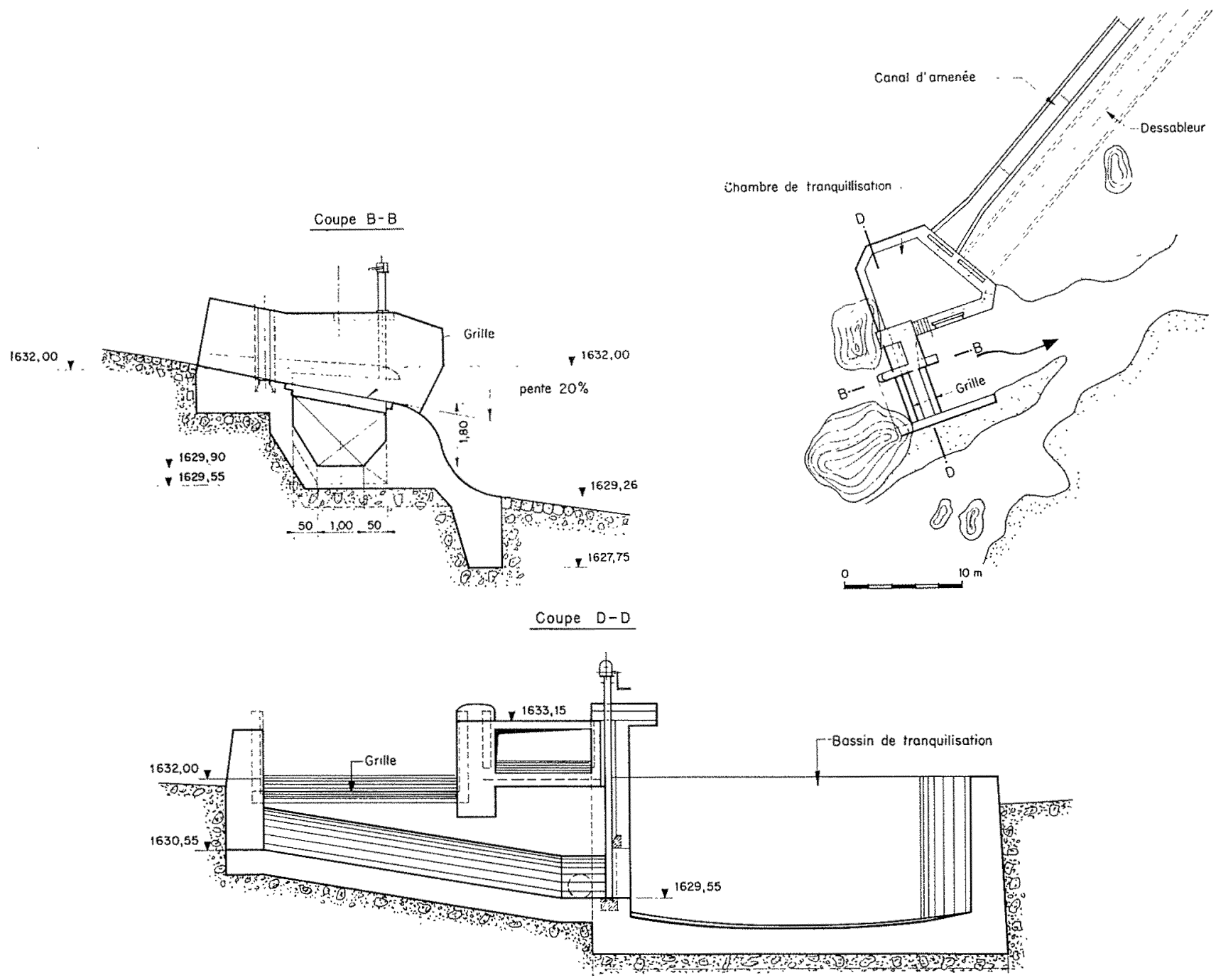

Fig. 5

Prise de Sengbach. 
contre, les grilles des prises d'été placées dans la tranche supérieure sont assez fréquemment obstruées par de petites branchilles;

...- La qualité du béton, si importante pour la longévité des ouvrages minces en montagne, est très bonne, comme le prouve l'absence de tous suintements à l'intérieur du dessableur à travers la dalle de $60 \mathrm{~cm}$ qui forme les parements du déversoir.

Fait intéressant, la conduite d'amenée traverse la moraine partiellement en galerie, partiellement en tranchée profonde; les joints des tuyaux se sont bien comportés.

Dans la partie de la galerie en moraine, malgré la présence d'une armature importante, des fissurations ont nécessité, lors de la mise en eau, l'application d'un enduit au Sika dont la tenue est bonne jusqu'à maintenant.

\section{c) Prise du Sengbach (fig. 5).}

Cette prise a été construite dans un délai extrêmement court: mise en chantier en mai 1958 , après la fonte des neiges, elle fut mise en scrvice la même année au début d'octobre.

Les raisons suivantes ont milité en faveur du choix d'une prise du type «en dessous», (dite aussi tyrolienne) :

- forte pente du torrent (de l'ordre de $20 \%$ );

- impossibilité d'implanter un petit barrage de retenue sur la roche en place,

- nécessité d'évacuer les matériaux charriés en utilisant la force vive du torrent,

- simplicité, insensibilité à l'action de crue très brutales,

- accès malaisé, nécessitant une réduction du volume des matériaux de construction à transporter.

Les conditions topographiques laissaient une grande liberté pour le niveau d'implantation de la prise, si bien que l'on a pu profiter de la présence de deux énormes blocs rocheux pour constituer les bajoyers naturels de la prise.

Celle-ci se compose d'un canal trapézoïdal muni d'une grille d'entrée de $2 \times 4 \mathrm{~m}$, d'un bassin de tranquillisation muni d'une purge manœuvrée à la main, avec un déversoir latéral limitant le débit capté en cas de crue.

Pour effectuer les réparations, de petits batardeaux peuvent être mis en place en amont de la grille; dans ce cas, le torrent s'écoule sur un coursier latéral formant déversoir.

La grille a été calculée en admettant une obstruction de $50 \%$ de la surface. Les barreaux trapézoïdaux, s'amincissant vers le bas, sont lar- ges de $20 \mathrm{~mm}$ en haut avec un coefficient de vide de $33 \%$. Mécaniquement, les barreaux sont largement surdimensionnés pour résister au choc lors du passage des blocs.

Pour le moment, il n'a pas été construit de dessableur, mais il est prévu de pouvoir l'ajouter ultérieurement selon les expériences faites concernant l'importance et la composition des matériaux décantés dans le bassin dépotoir.

De toute façon, il est prévu de mettre la prise hors service lorsque le débit du Sengbach est supérieur à $2 \mathrm{~m}^{3} / \mathrm{s}$. Dans ce cas, les autres prises suffisent à assurer seules le fonctionnement à pleine charge de la centrale.

Enfin, on espérait que, grâce à l'emploi d'agrégats siliceux, l'usure de la conduite d'amenée dans les zones à grande vitesse ne serait pas trop rapide.

L'expérience a démenti cette hypothèse; en effet, certaines parties de la conduite d'amenée en tuyaux de béton, probablement soumises à des écoulements perturbés, présentent déjà une usure des parois de 5 à $10 \mathrm{~mm}$ après deux ans d'exploitation.

En cas de crue exceptionnelle, les débits de torrent peuvent passer dans un bras secondaire, par une brèche du mur d'endiguement du torrent. Enfin des blocs ont été enchâssés dans le radier à l'amont de la prise pour diminuer la vitesse de passage sur la grille.

Pour la conduite d'amenée, Iongue de $1 \mathrm{~km}$ environ, entre la prise et le bassin d'Eggen, on a recherché une solution permettant d'éviter les excavations dans l'éboulement formé d'énormes blocs et garantissant la tenue au gel d'un ouvrage extérieur. Pour le tronçon supérieur de $220 \mathrm{~m}$ qui traverse l'éboulement, on s'est arrêté à un chenal à écoulement libre, composé d'éléments trapézoïdaux préfabriqués et précontraints de $4 \mathrm{~m}$ de long, d'un poids de $2400 \mathrm{~kg}$, reposant sur des sellettes transversales placées aux joints. Les éléments de chenal sont assez légers poux être mis en place au moyen d'un téléphérique de chantier rudimentaire et peu coûteux, construit par un entrepreneur local.

Les joints (joints Ott), points faibles de toute construction de cette nature, ont été réalisés de façon astucieuse (fig. 6).

On a évité toute battue en bout des éléments de chenal. Sur les deux lèvres d'extrémité, séparées par un espace de 2 à $3 \mathrm{~cm}$, deux bandes de caoutchouc sont collées au béton. Le couvrejoint métallique, dont la section transversale ressort de la figure 6 , enveloppe et protège complètement les garnitures de caoutchouc. Il est serré au moyen de tiges filetées munies de contreplaques qui prennent appui directement sur la face extérieure du béton. Ainsi les éléments peuvent jouer l'un par rapport à l'autre. 


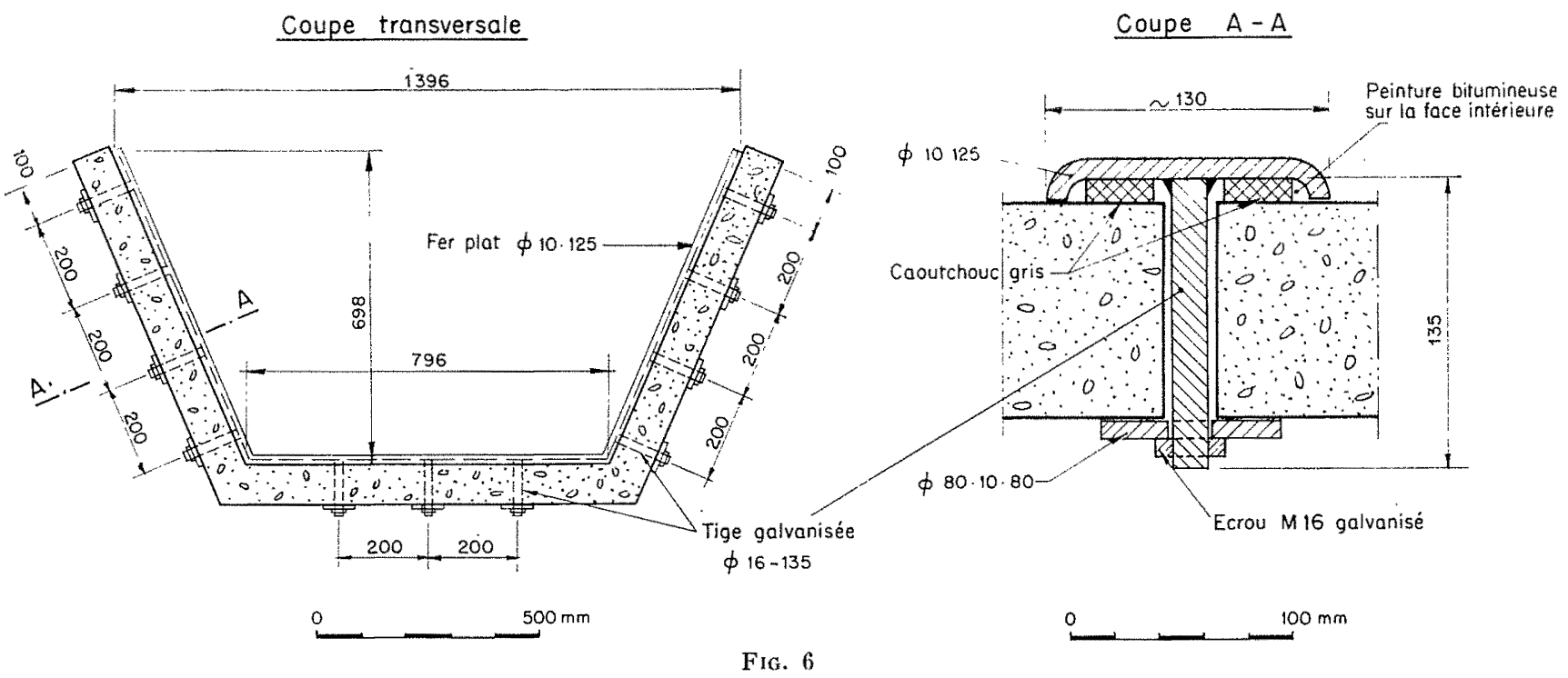

Joint ott entre des éléments préfabriqués précontraints du canal à écoulement libre.

La présence de ces couvre-joints ne perturbe pas sensiblement l'écoulement.

Il a été observé en exploitation que :

- les ouvrages de prise ont bien supporté les crues, notamment une importante crue en automne 1958 (estimée à $15 \mathrm{~m}^{3} / \mathrm{s}$ ),

- la grille ne s'est jamais obstruée,

- les blocs enchâssés dans le radier ont bien résisté,
- en hiver, la grille gèle, mais l'eau passe sous la glace, même lorsque la température est très basse,

- durant la débâcle printanière, les matériaux décantés atteignent 3 à $6 \mathrm{~m}^{3}$ par jour, et il faut purger journellement,

- par contre, l'entretien de la prise exige de la part du gardien un soin attentif et des interventions fréquentes pour purger,

- les joints du chenal d'arrivée se sont bien comportés : ils sont parfaitement étanches.

\section{CONCLUSION}

L'aménagement de Gabi présente des prises de divers types, adaptés aux conditions hydrologiques et morphologiques particulières dans chaque cas.

Aux trois prises d'Eggen sur le Krummbach, Alpjen sur le torrent du même nom, et sur le Sengbach en service respectivement depuis trois et deux ans, on a pu faire les constatations suivantes :

\section{Prise-BarRage d'Eggen :}

La prise d'Eggen est du type latéral; dans ce genre de prise on recherche essentiellement par le choix de l'implantation sur le cours d'eau, par les formes hydrauliques appropriées, par le calage du seuil de prise, à empêcher la pénétration des matériaux grossiers charriés par le cours d'eau. Le dessableur subséquent n'a pas d'autre rôle à jouer que de retenir les matériaux en suspension. Dans le cas particulier, le dessableur a été réduit pour ne retenir que des éléments moyens, vu la présence d'un bassin de compensation, mais il a été muni à titre d'essai de dispositifs d'évacuation automatiques brevetés «Gianella». Ce système fonctionne bien et présente une amélioration vis-à-vis du dispositif classique avec gouttière à purge ouverte.

La vanne de purge spéciale également brevetée fonctionne sans défaillance; par contre, la commande automatique par contrepoids hydraulique n'est pas assez puissante et pourrait être remplacée par un système à relais hydrauliques, tel qu'il a été adopté par l'EdF. 
Рното 6

Prise d'Alpjen.

Vue générale amont à gauche.

Remarquer

le barrage triangulaire

contenant le dessableur.

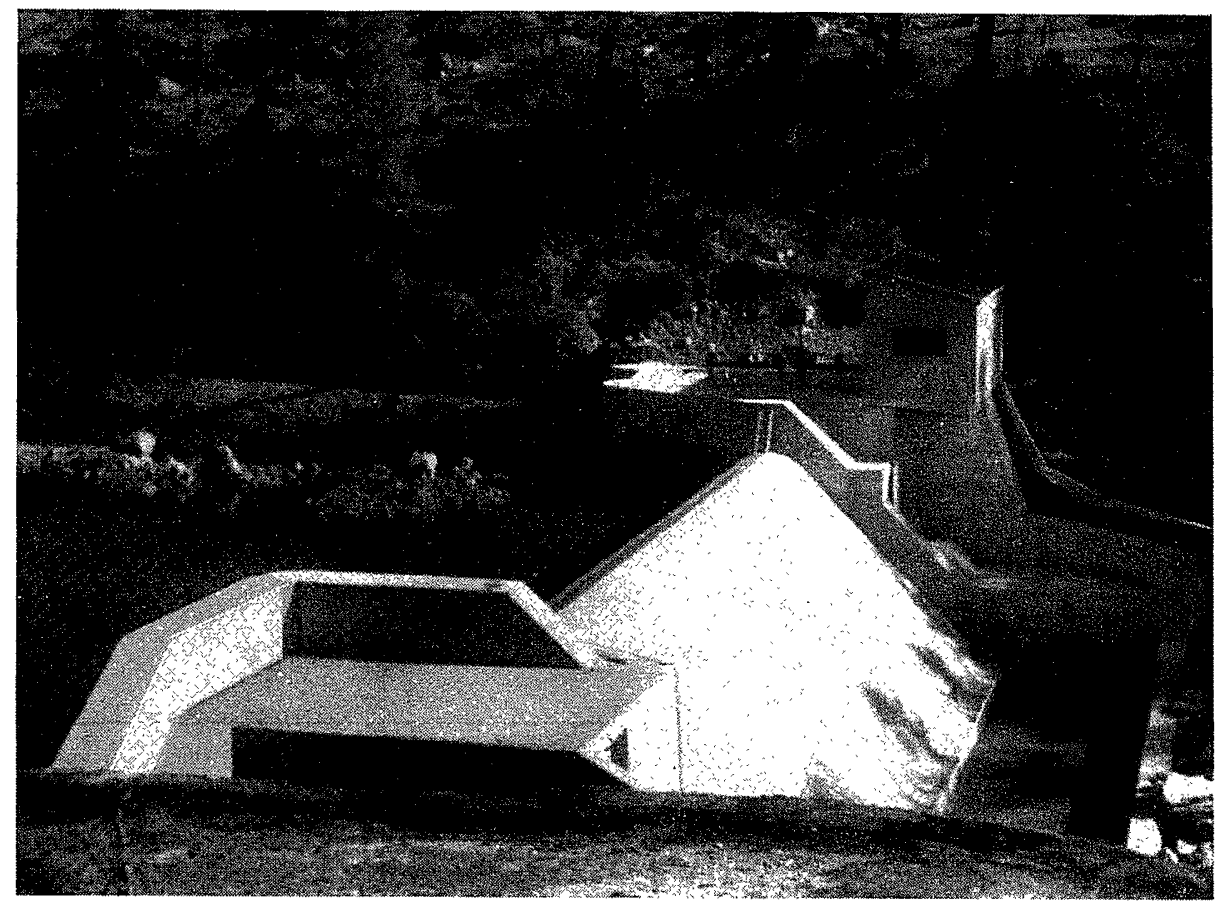

Les possibilités d'évacuation des sables et graviers sont telles qu'on pourrait, dans une certaine mesure, attacher une importance moins grande aux sujétions hydrauliques mentionnées ci-dessus.

\section{Prise D'AlpJen :}

La prise d'Alpjen est caractérisée par une disposition du barrage de prise, évidé, contenant le dessableur et comportant un très petit volume de béton dans une région escarpée où les transports sont particulièrement coûteux.

Le débit de prise est réglé sans organe mécanique, de façon à le rendre pratiquement indépendant de la hauteur de la lame déversante en cas de crue, ce qui a l'avantage d'introduire un minimum de matériaux en suspension puisque, contrairement à la prise d'Eggen, la prise d'Alpjen alimente directement les turbines de Gabi sans bassin intermédiaire.

La commande de la purge du dessableur n'est dans ce cas pas automatique.

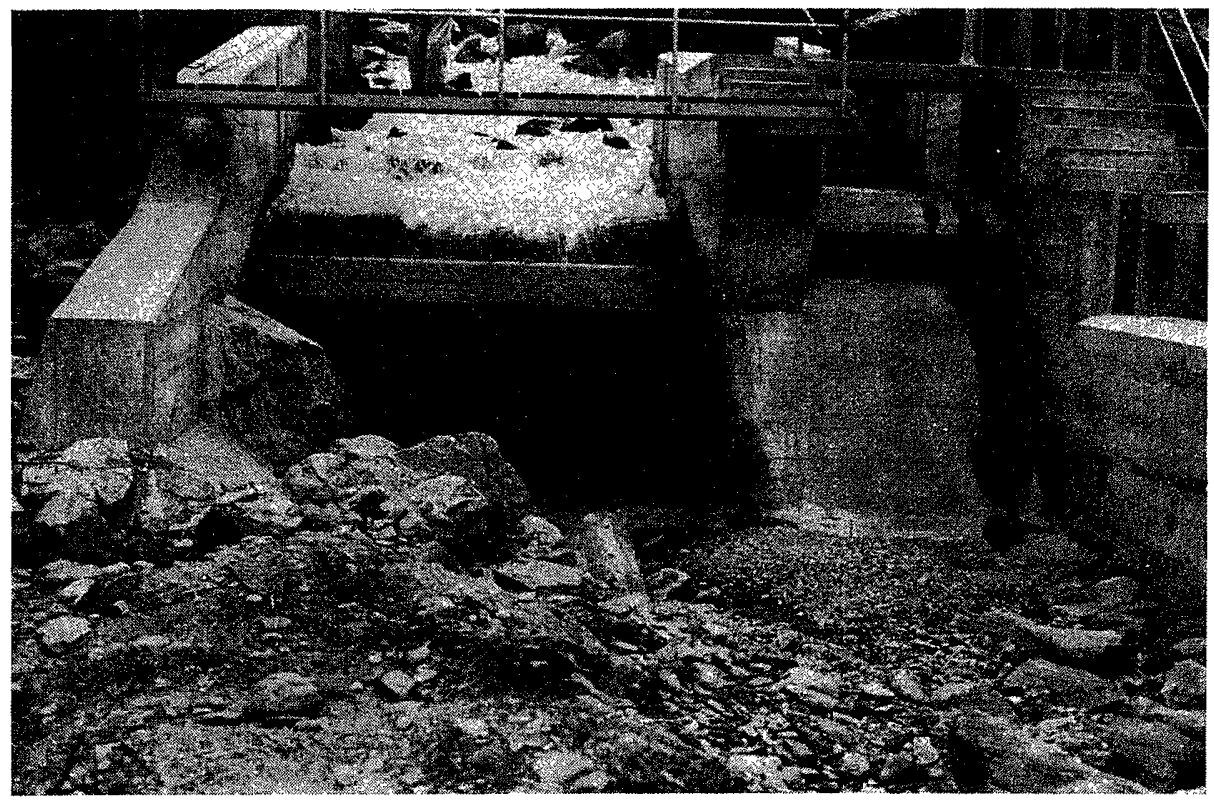

Pното 7

Prise \&tyrolienne» (par en-dessous) du Sengbach. 


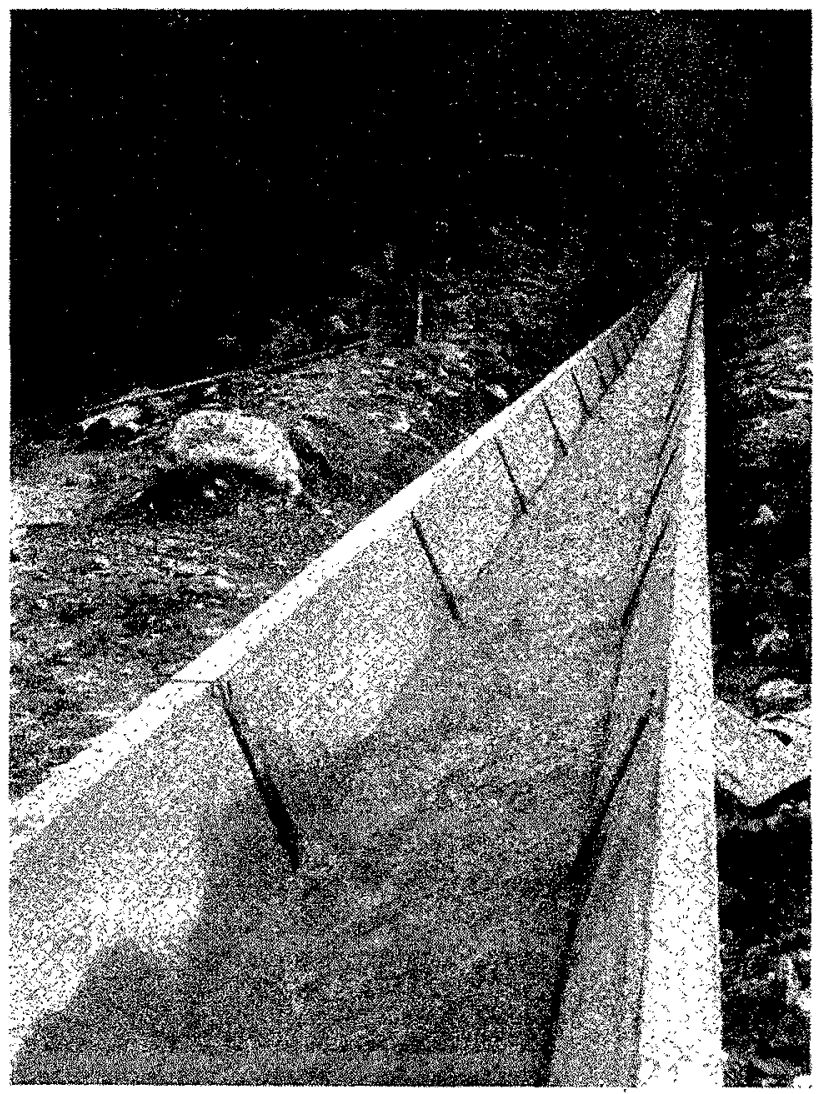

Proto 8

Chenal préfabriqué en béton. Remarquer le fable sillage provoqué par les joints métallques en saillie.

Prisfe du Srivgagh :

La prise du Sengbach est une prise tyrolienne ( en dessous s) sur un torrent glaciaire sauvage aux eaux tròs chargées en été. Cette prise est mise hors service en période de grosses fontes, lorsque les autres prises suffisent à alimenter l'usine. Pour cette raison, elle ne comporte pas de dessableur, mais un simple dépotoir commandé à main. L'expérience a montré qu'il n'est pas difficile d'évacuer les matériaux fins que cette prise amene dans le bassin de compensaLion, mais un dessableur aurait l'avantage d'empêcher les usures trop rapides de certaines parties de la conduite d'amenée qui présente des vitesses jusqu'à $4,5 \mathrm{~m} / \mathrm{s}$.

Par la suite des formes bien étudiées, il ne se produit pas d'obstruction de la grille, ni en été par les sédiments, ni en hiver par la glace.

Dans cette prise, l'écartement des barreaux est serré, les dispositifs de dégravage automatique développés depuis lors, notamment par l'EdF, Alpes-II, permettent d'augmenter l'écartement des barreanx et de les faire massifs, ce qui supprime absolument tout risque d'obstruction et confère a la prise tyrolienne des avantages tels que sa diffusion se généralise de plus en plus.

\section{CONDUITE D'AMENÉE :}

Un troncon à écoulement libre réalisé en éléments préfabriqués précontraints a permis d'écarter les sujétions de la préparation et de la mise en place de beton et d'obtenir un ouvrage resistant au gel.

Le joint $O \mathrm{tt}$, d'un type nouveau, a permis de supprimer les déficiences généralement constatées dans les joints encastrés.

$$
*
$$

II est souvent regrettable qu'après la mise en exploitation d'ouvrages de cette nature, les améliorations de détails ne puissent plus être entreprises. Il suffit en effet bien souvent d'un peti! complément de dépenses pour apporter des améliorations déterminantes, suite des observations faites an cours des premières années d'exploitation. Le bon fonctionnement des ouvrages, notamment des prises d'eau, dépend de l'étroite collaboration entre projeteurs et exploitants qui doit se poursuivre au-dela de la mise en exploitation.

Nous remercions l'Energie Electrique du Simplon d'avoir bien voulu nous autoriser à communiquer les expériences faites à l'occasion de la construction et de l'exploitation de ses aménagements, dont l'étude et la direction des travaux ont été assumées par la Société Généràle pour l'Industrie à Genève.

Nous nous plaisons à citer ici nos collaborateurs, en particulier MM. Gardet et Hatt t, qui ont participé à la conception de ces ouvrages.

\section{ERRATUM}

Dans notre numéro 2, mars-avril 1961, page 161, colonne 2 , au renvoì de bas cle page $n^{\circ} 3$ bis, lire :

Conduites forcées de l'usme de Gémissiat $\theta=28$ (au lieu de 2,8). 